\title{
Invited Review Article: Accurate and fast nanopositioning with piezoelectric tube scanners: Emerging trends and future challenges
}

\author{
S. O. Reza Moheimani ${ }^{\text {a) }}$ \\ School of Electrical Engineering and Computer Science, The University of Newcastle, University Drive, \\ New South Wales 2308, Australia
}

(Received 1 April 2008; accepted 21 June 2008; published online 21 July 2008)

\begin{abstract}
Piezoelectric tube scanners have emerged as the most widely used nanopositioning technology in modern scanning probe microscopes. Despite their impressive properties, their fast and accurate operations are hindered due to complications such as scan induced mechanical vibrations, hysteresis nonlinearity, creep, and thermal drift. This paper presents an overview of emerging innovative solutions inspired from recent advances in fields such as smart structures, feedback control, and advanced estimation aimed at maximizing positioning precision and bandwidth of piezoelectric tube scanners. The paper presents a thorough survey of the related literature and contains suggestions for future research prospects. (C) 2008 American Institute of Physics. [DOI: 10.1063/1.2957649]
\end{abstract}

\section{INTRODUCTION}

Nanotechnology is the science of understanding and control of matter at dimensions of $100 \mathrm{~nm}$ or less. A key enabling tool for nanotechnology research is scanning probe microscopy. This family of microscopes allows researchers to study and manipulate matter at the molecular and atomic levels. Central to the operation of a scanning probe microscope (SPM) is a scanning device that is required to operate with nanometer or subnanometer precision. A number of technologies have been proposed to realize such a mechanism. At the time of this writing, piezoelectric tube scanners are the most widely used scanning technology in SPMs. Positioning accuracy and speed of a piezoelectric tube scanner are limited due to certain complications inherent to piezoelectric materials. This article describes a number of emerging trends aimed at realizing fast and accurate scanning mechanisms for SPMs.

The paper is structured as follows. Section II presents a very brief introduction to scanning probe microscopy, emphasizing the need for rastering. Various rastering technologies, including piezoelectric tube scanners, are discussed in this section. Section III is concerned with electromechanical properties of piezoelectric tube scanners. The $x-y-z$ positioning capability and the resonant nature of the scanner and its adverse effect on the achievable scanning speed are discussed. The effects of hysteresis, creep, and thermal drift on the positioning accuracy of a tube are explained, and the possibility of utilizing feedback control as a remedy is explored. Section IV concentrates on electrical properties of a piezoelectric tube scanner. In particular, a piezoelectric tube actuator is modeled as a two-port electric circuit. The possibility of using strain-induced piezoelectric voltage as a measurement signal in a feedback loop is investigated in this section. It is explained how this signal can be utilized to improve the displacement measurements obtained from a ca-

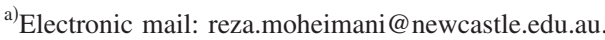

pacitive sensor. Section IV discusses the sensorless control of a piezoelectric tube scanner and reveals its inherent feedback structure. Section V discusses possible future directions and concludes the paper.

\section{SCANNING PROBE MICROSCOPY}

Scanning probe microscopy refers to a family of instruments that enables us to study and also to manipulate matter over the dimensions of several $100 \mu \mathrm{m}$ to $10 \mathrm{pm}$. SPMs are therefore capable of interrogating and altering the surface profile of a sample at the molecular and atomic levels. The two most widely used SPMs are scanning tunneling microscopes (STMs) and atomic force microscopes (AFMs). The STM was invented in early 1980s by Binnig and Rohrer at the IBM Zurich Research Laboratory in Switzerland. ${ }^{1,2}$ It uses the principle of electron tunneling ${ }^{3}$ between a sharp probe tip and a conducting surface to develop a threedimensional (3D) image of the surface with atomic resolution. The probe is brought to within a nanometer distance of the surface, and a potential difference is introduced between the two objects. Once a tunneling current is established, the probe is moved in a raster pattern in the $x-y$ plane. The distance between the probe and the surface is adjusted during rastering to regulate the current. Plotting the vertical movements of the probe against its $x-y$ coordinates results in a topographical image of the surface with a very high resolution.

Like the STM, the AFM relies on a scanning approach to produce 3D images of material surfaces with extremely high resolutions. The AFM measures ultrasmall forces (in the order of $1 \mathrm{nN}$ or less) between the AFM tip and a sample surface. ${ }^{4}$ The force is determined by measuring the motion of a very flexible microcantilever with an extremely small mass. A key advantage of the AFM is its ability to investigate surfaces of nonconducting as well as conducting materials, thus overcoming a major restriction of STM. Good reviews of scanning tunneling and atomic force microscopy can be found in Refs. 5-8. 
An important component of a SPM is its scanning head that moves either the sample or the probe in a raster pattern in the $x-y$ plane. It also controls the distance between the probe and the sample through the $z$-axis servo. A number of technologies have been proposed to make this functionality feasible. The first STMs used piezoelectric tripod scanners for rastering. ${ }^{2}$ In this arrangement three piezoelectric stack actuators are used to form a tripod. A probe is attached to the tip of the tripod where its three legs meet. The $x-y$ rastering and the $z$-axis movements are achieved by applying appropriate signals to the three stack actuators. To achieve an acceptable scanning range, a tripod scanner would have to be built to be relatively large. This arrangement can be rather bulky and is, thus, rarely used in modern SPMs.

The use of a piezoelectric tube actuator with quartered electrodes for 3D positioning in a SPM was first reported in Ref. 9. Today, the majority of commercially available SPMs use a piezoelectric tube scanner for $x-y-z$ positioning. They have a simple construction, are easy to integrate into a microscope, and are relatively inexpensive.

Flexure-based 2D and 3D nanopositioning platforms have recently been incorporated into a small number of commercially available AFMs. ${ }^{10}$ These positioning stages typically have a parallel kinematic design and use piezoelectric stacks for actuation and a flexure guidance system to eliminate friction and stiction. ${ }^{11,12}$ Small motions generated by piezoelectric stack actuators are amplified using a mechanical leveraging system. Capacitive sensors are integrated within these nanopositioning platforms making it possible to use advanced control design methodologies to increase their precision and bandwidth. ${ }^{13-15}$ These scanners are particularly attractive due to the low presence of cross-coupling between their various axes. In terms of scanning speeds, flexure-based scanners are comparable with piezoelectric tube scanners for a relatively similar scanning range. An experimental scanning system proposed recently in Refs. 16 and 17 has been shown to have the potential to achieve very fast scanning speeds. However, due to its direct drive nature, its scanning range is rather limited.

Compared with piezoelectric tube scanners, flexurebased positioning platforms are much more complicated mechanisms and hence, at the time of this writing, substantially more expensive. Consequently, piezoelectric tube scanners are likely to remain the most widely used positioning platform in SPMs and in other applications where mirco- and nanoscale positioning is a necessity. With regard to the latter, we may refer to applications such as piezoelectric tube-based gyroscopes, ${ }^{18,19}$ inertial drives, ${ }^{20}$ and cell manipulation instrumentation. ${ }^{21}$

\section{ELECTROMECHANICAL PROPERTIES OF A PIEZOELECTRIC TUBE SCANNER}

\section{A. Mechanical construction}

As illustrated in Fig. 1, a piezoelectric tube scanner comprises a tube of radially poled piezoelectric material, four external electrodes, and an internal electrode. One end of the tube is fixed and the other end is free to move. Opposite external electrodes are segmented in four equal sectors of
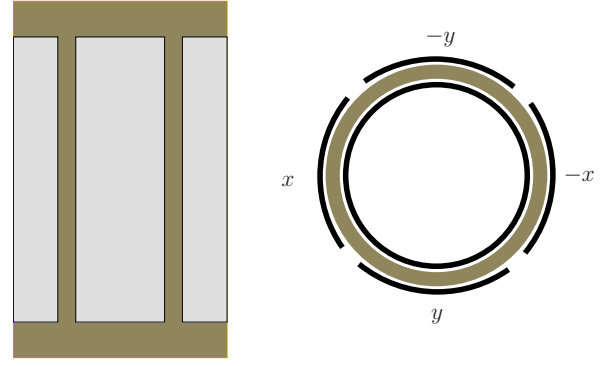

FIG. 1. (Color online) Side view and top view of a piezoelectric tube scanner with quartered external electrodes and a continuous inner electrode.

$90^{\circ}$. Opposite electrodes are driven by signals of identical magnitudes but opposite signs, as illustrated in Fig. 2(a). Once a voltage is applied to these electrodes, one side of the tube extends vertically, while the opposite side contracts, resulting in bending. This, in turn, results in a two-dimensional movement of the free end of the tube in the $x-y$ plane. For $x-y$ actuation, the inner electrode is grounded. To actuate the tube in the $z$ direction, however, the inner electrode is driven by the $z$ signal. An appropriate application of the three signals allows for full $x-y-z$ positioning of the free end of the tube.

Another configuration includes a circumferential outer electrode for independent vertical extension or contraction. A drawback of this configuration is that the $x-y-z$ scan range may be reduced as the electrodes on the outer surface of the

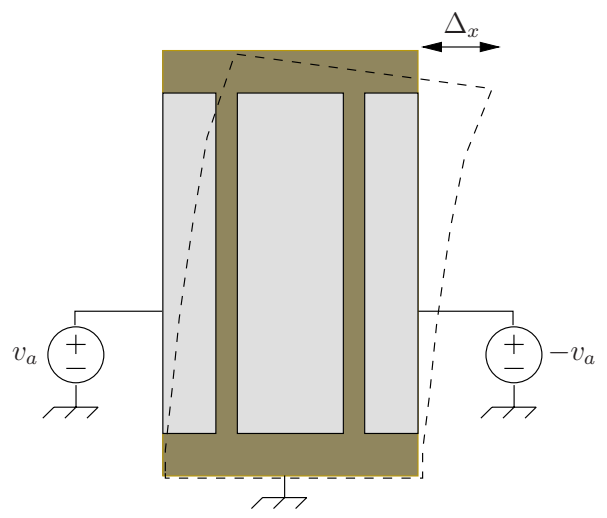

(a)

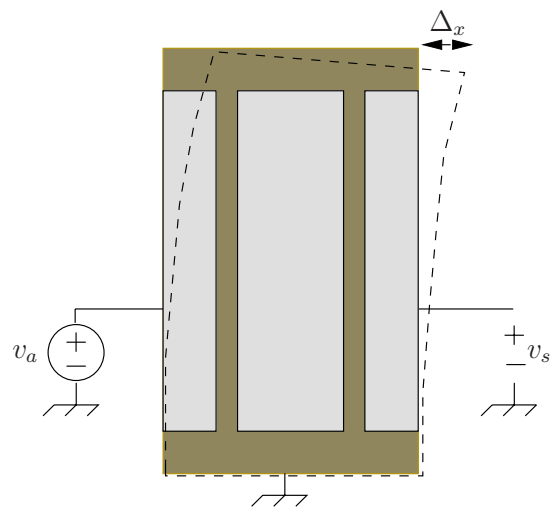

(b)

FIG. 2. (Color online) Sensorless control of a piezoelectric tube scanner: (a) strain voltage induced in one electrode is used for feedback; (b) one of the electrodes is shunted to an impedance. 
tube are used for actuation in all three directions. An advantage is that the inner electrode is grounded at all times, simplifying the task of designing electronic amplifiers that are used to drive the actuator. Yet another possibility is to have quartered internal electrodes. For the remainder of this review, we assume that the tube electrodes are as shown in Fig. 1.

\section{B. Mechanical deflection}

A precise calculation of the scanning range of a piezoelectric tube could be rather difficult. ${ }^{22-24}$ The bending of the tube is a function of the electric field and the nonuniform induced strain. However, assuming that opposite voltages are applied to the opposite electrodes, as in Fig. 2(a), and assuming small deflections would result in the following approximate expression for the tip translation along the $x$ or $y$ direction: ${ }^{24}$

$$
\Delta_{i}=\frac{2 \sqrt{2} d_{31} L^{2}}{\pi D h} v_{i}, \quad i=x, y .
$$

Here, $\Delta_{i}$ is the deflection in the $x$ or $y$ direction, $d_{31}$ is the piezoelectric strain constant, $L$ is the tube length, $D$ is the outside diameter of the tube, $h$ is the tube thickness, and $v_{i}$ represents the $x$ - or $y$-axis electrode voltage.

Alternatively, one may choose to apply the actuation voltage only to one of the two electrodes, as illustrated in Fig. 2(b). This would halve the tip deflection expressed in Eq. (1). Incidentally, this is the arrangement used in the original design of Binnig and Smith reported in Ref. 9. When operated in this mode, the opposite electrode is often grounded. However, instead of grounding this electrode, it is possible to use the voltage induced in it due to the piezoelectric effect for other purposes. This property of piezoelectric tube scanners has been overlooked by researchers and will be discussed in detail later in Sec. IV.

Vertical translation of the tube due to a voltage $v_{z}$ applied to its inner electrode is approximately given by

$$
\Delta_{z}=\frac{d_{31} L}{h} v_{z} \text {. }
$$

\section{Resonant nature}

Static deflections of a tube expressed in Eqs. (1) and (2) remain valid at low frequencies. To understand the dynamics of a tube at higher frequencies requires a detailed finite element analysis. ${ }^{22,25,26}$ It turns out that a piezoelectric tube scanner is a highly resonant system. Consequently, the transfer function of a tube possesses numerous lightly damped poles, i.e., poles that are very close to the $j \omega$-axis. The frequency response of a tube is illustrated in Fig. 3. This particular piezoelectric tube is made from a PZT-5H piezoelectric ceramic material. Its height is $63.5 \mathrm{~mm}$, its thickness is $0.66 \mathrm{~mm}$, and its outer diameter is $9.52 \mathrm{~mm}$. Figure 3 plots the lateral displacement of the tube, measured by a capacitive sensor, as a result of a signal applied to the opposite electrode. A signal that contains frequency components at or
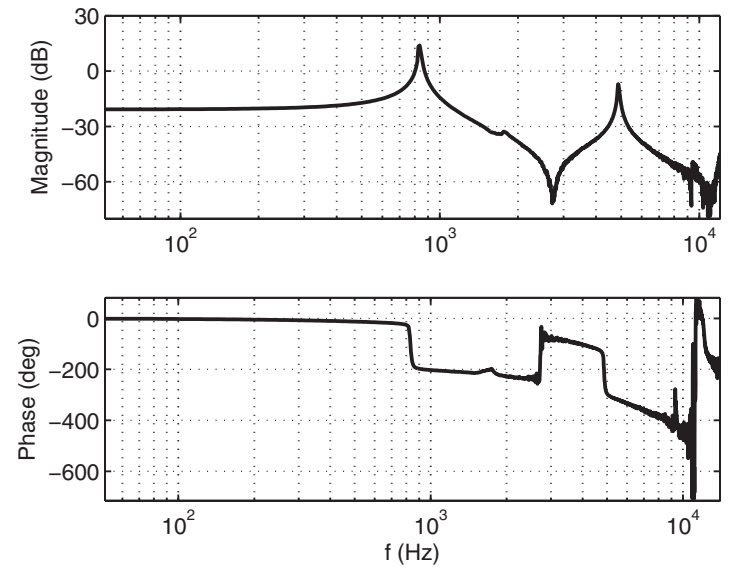

FIG. 3. Frequency response of a piezoelectric tube scanner with quartered external electrodes and a single inner electrode. The arrangement is similar to Fig. 2(b). One of the electrodes is taken as the input. The lateral deflection of the tube was measured by a noncontact capacitive sensor with a bandwidth of $10 \mathrm{kHz}$. The apparent phase roll-off is due to the presence of a second order Butterworth low-pass filter in the capacitive sensor.

close to the tube's first resonance frequency will result in significant displacements. This leads to a major complication in high-speed operations of SPMs.

To force the free end of the tube to move in a raster pattern, a triangular signal is applied to the $x$-axis (fast axis) electrodes and a slowly increasing ramp signal is applied to the $y$-axis (slow axis) electrodes. This is depicted in Fig. 4, where a pseudoramp signal is applied to the slow axis. A triangular waveform contains all odd harmonics of the fundamental frequency. Although the amplitudes of these harmonic signals diminish as $1 / n^{2}$, with $n$ being the harmonic

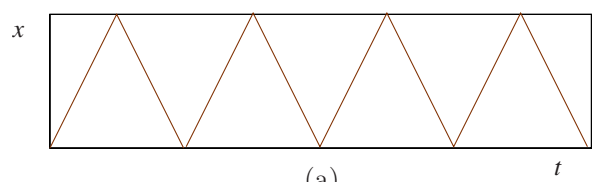

(a)

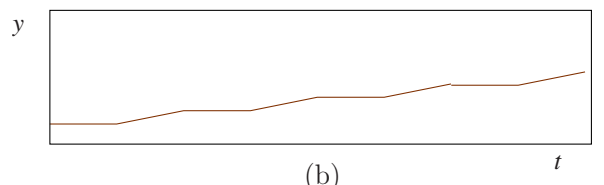

(b)

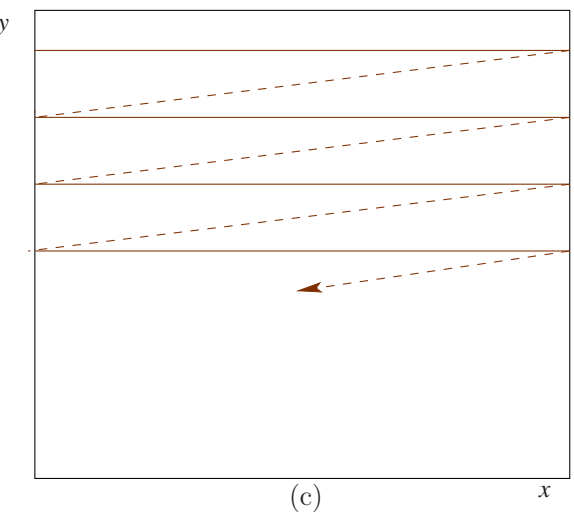

FIG. 4. (Color online) In order to force the scanner to trace a raster pattern (c) in the $x-y$ plane, a triangular signal (a) is applied to the fast axis and a psuedoramp signal (b) is applied to the slow axis. 


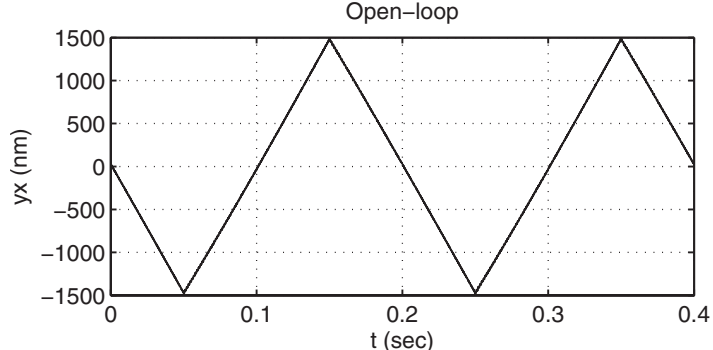

(a)

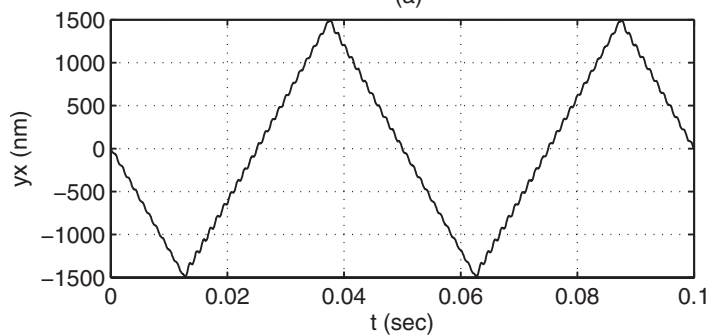

(b)

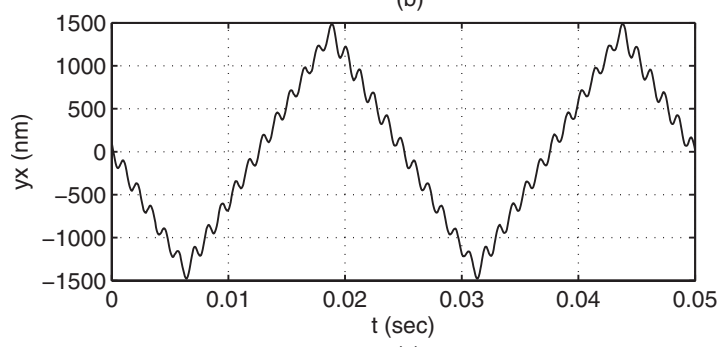

(c)

FIG. 5. Open-loop lateral movement of a piezoelectric tube scanner when driven by (a) $5 \mathrm{~Hz}$, (b) $20 \mathrm{~Hz}$, and (c) $40 \mathrm{~Hz}$ signals. The actuator was driven by a charge source, and thus no sign of hysteresis can be observed in these plots.

number, ${ }^{27}$ if a fast triangular waveform is applied to the tube, it will inevitably excite the resonance. This situation is depicted in Fig. 5. The 21st harmonic of the $40 \mathrm{~Hz}$ signal is relatively close to the first resonance frequency of the tube, i.e., $850 \mathrm{~Hz}$. Although its corresponding amplitude is more than 440 times smaller than the $40 \mathrm{~Hz}$ component of the triangular signal, and despite the fact that it is $10 \mathrm{~Hz}$ away from the resonance, this harmonic generates significant distortions. Thus, instead of following a perfect triangle, the free end of the tube traces a distorted triangular waveform along the fast axis. Consequently, the resulting raster pattern will be distorted. To avoid this complication, the scanning speed of SPMs is often limited to about $1 \%$ of the tube's first resonance frequency. For the tube whose frequency response is illustrated in Fig. 3, this amounts to a speed limitation of about $8 \mathrm{~Hz}$.

\section{Hysteresis, creep, and thermal drift}

Piezoelectric materials exhibit a hysteretic behavior. Thus, a piezoelectric actuator driven by a voltage amplifier is a nonlinear device and should be treated as such. Due to the presence of hysteresis, when a triangular voltage signal is applied to a piezoelectric actuator, the resulting displacement can deviate from linear by as much as $15 \%$ between the forward and backward movements. The commercially available SPM software usually compensates for the hysteresis effect by scanning always in the same direction. ${ }^{28}$ In this open-
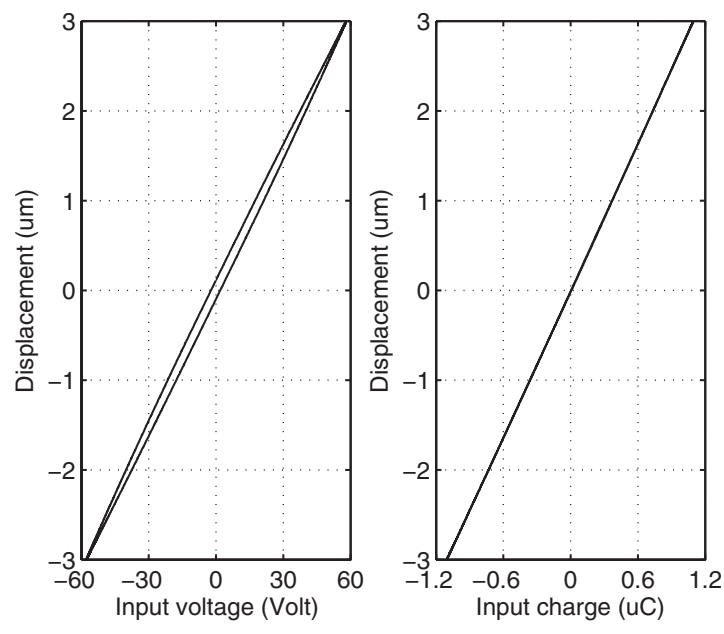

FIG. 6. Hysteresis plots of a piezoelectric tube scanner driven by voltage and charge signals. The use of a charge source significantly diminishes the presence of nonlinearity.

loop approach, the input triangular signal is perturbed to achieve an acceptable trajectory, thus minimizing the effect of this particular form of nonlinearity. Another possibility is to model a piezoelectric actuator as a linear dynamic system cascaded with a static nonlinearity that represents the hysteretic nature of the device. A number of techniques have been proposed to model hysteresis in this framework, including the classical Preisach hysteresis model ${ }^{29-32}$ and the Maxwell resistive capacitor model. ${ }^{30,33}$ Feedback-based techniques such as adaptive control ${ }^{34}$ and robust control ${ }^{15,35}$ design techniques have also been used to deal with hysteresis in nanoscale positioning platforms. Another method that does not require an accurate model is the phaser approach reported in Refs. 36 and 37. Describing functions have also been implemented with some success. 38,39

An interesting and unique property of piezoelectric materials is that when driven by a charge source, they display minimal hysteresis. Figure 6 illustrates the improvement that can be achieved when a piezoelectric tube scanner is controlled by a charge source. This property has been known since 1980 s. $^{40,41}$ However, until very recently, it has been rarely used due to the difficulties associated with driving highly capacitive loads with commercially available charge or current amplifiers. The key problem is that the output signal produced by such amplifiers when applied to a piezoelectric actuator tends to drift with time due to the highly capacitive nature of the load. This issue has been observed by a number of researchers. ${ }^{38,42}$ Recent research $^{43,44}$ proposes a new structure for charge and current sources capable of regulating the dc profile of the actuator. A dc-accurate charge/current amplifier has also been reported in Refs. 45 and 46. Other recent studies in this area include Ref. 47 and 48.

Creep is another undesirable property common in piezoelectric materials. When the applied voltage to a piezoelectric actuator undergoes a sudden change, the piezoelectric material will creep, and this can result in significant loss in precision when positioning is required over extended periods of time. ${ }^{49}$ In particular during slow operation of SPMs, creep can result in significant distortions in the generated image. ${ }^{50}$ 
In particular, creep has two adverse effects in SPMs: (i) the vertical position of the tip will creep after it approaches the sample, and (ii) it exacerbates the effect of hysteresis at the turning point of the scanning trajectory. A number of methods have been proposed to deal with this phenomenon. One approach is based on approximating the creep effect as a nonlinearity that can then be inverted, ${ }^{14,51}$ while another method, presented in Ref. 52, develops a linear model to describe the low-frequency nature of creep. In fast scanning probe microscopy, creep is of little consequence. However, due to the fact that most SPMs are operated quite slowly, its effect cannot be overlooked.

Although scanning probe microscopy was originally invented as an imaging tool, it is being increasingly used as a nanoscale manipulation device. ${ }^{53-57}$ One of the most vexatious features of SPMs, particularly in applications that involve nanomanipulation, is the thermal drift of their mechanical setup. This arises from the thermal expansion and contraction of their mechanical components. In a typical AFM, operated in ambient temperature, a $1^{\circ}$ change in temperature can cause a $50 \mathrm{~nm}$ drift. ${ }^{58}$ Although this effect can be suppressed in low temperature experiments (mainly in UHV SPMs), in applications that involve interrogation and manipulation of the matter in ambient temperature it amounts to a substantial hurdle. A number of techniques have been proposed to compensate for this effect, e.g., Refs. 59-63. Recent research ${ }^{58}$ shows that a Kalman filter can be used to estimate the drift quite effectively, and subsequently compensate for it.

\section{E. Feedback}

There has been a consistent effort in recent years to improve accuracy and speed of SPMs using feedback control techniques. One of the earliest attempts to control a piezoelectric tube scanner is reported in Ref. 64, where a noncontacting inductive sensor was used to measure the lateral displacement of a tube. Lag lead and $H_{\infty}$ controllers were designed and implemented on the tube, and the feasibility of reducing the adverse effects of creep and hysteresis was demonstrated. The authors also reported that their $H_{\infty}$ controller achieved damping of high-frequency vibrations. Reference 65 describes the use of a loop shaping procedure to design a feedback controller for a piezoelectric tube actuator instrumented with optical displacement sensors to track a raster pattern. Their results show reductions in tracking error and cross-coupling due to the use of feedback control. Examples of other works showing successful applications of feedback include Refs. 66-68.

The key idea associated with feedback-based methods is to damp the first resonant mode of the piezoelectric tube actuator. This "flattening" of the frequency response of the scanner will allow tracking of a faster triangular waveform, and consequently a faster scan. Furthermore, in order to achieve accurate positioning at high frequencies, and to minimize the adverse effect of hysteresis, the feedback gain is often chosen to be high. There is a limit on how high the feedback gain can be made before the closed loop system is made unstable since the existence of sharp resonant peaks in the frequency response of the actuator typically results in a very low gain margin. ${ }^{69}$ Using notch filters in the feedback loop has been shown to result in an improvement in the achievable gain margin. ${ }^{70}$ The use of high gain feedback for accurate tracking is necessitated due to the hysteretic nature of the piezoelectric actuator. If the actuator is driven by a charge source, the hysteresis is significantly reduced, resulting in an almost linear system. Thus, using charge, one should expect to obtain a feedback control system that has better robustness properties.

An inversion-based feedforward method has also been applied to piezoelectric tube actuators. A feature of a feedforward control scheme is that it does not require any additional sensors for implementation. In Ref. 71, model-based inversion approach was used to compensate for positioning distortions caused by creep, hysteresis, and induced vibrations. A low-order feedforward controller was presented in Ref. 67 to suppress the lateral oscillation of a piezoelectric tube scanner. The feedforward controller was designed using the $H_{\infty}$ method such that the system is not excited at frequencies around the first resonance of the piezoelectric tube scanner. The performance of feedforward control schemes heavily relies on an accurate model of the system. ${ }^{67,71} \mathrm{Com}-$ bining feedback and feedforward compensations has been shown to result in satisfactory tracking in a piezoelectric tube nanopositioner in the presence of parameter uncertainties in the plant model. ${ }^{72}$

The key problem associated with using feedback control in piezoelectric tube scanners is the need for a sensor to measure displacement. Displacement sensors are often expensive, and their integration into the scanning stage of SPMs could be a difficult task. The main drawback of using displacement sensors, however, is noise. Capacitive sensors have emerged as the most widely used displacement sensors in SPMs. The capacitive sensor used to generate the frequency response in Fig. 3 can measure up to $100 \mu \mathrm{m}$ and has a root-mean-square (rms) noise of about $20 \mathrm{pm} / \sqrt{\mathrm{Hz}}$. Over a bandwidth of $10 \mathrm{kHz}$, this amounts to a rms noise of $2 \mathrm{~nm}$, clearly inadequate for atomic level manipulations and interrogations. Limiting the bandwidth to $1 \mathrm{~Hz}$ would reduce the sensor noise to a level that is sufficient for scanning tunneling microscopy, e.g., for atomic resolution on graphite. However, this would significantly limit the scanning speed of the device. Consequently, feedback has not been used in STMs.

If the presence of sensor noise can be tolerated, the use of feedback control in SPMs becomes a very attractive option. Apart from the (extremely important) possibility of achieving much higher scanning frequencies, feedback allows one to address other issues that hamper fast operation of SPMs. For example, it is common practice to ignore the cross-coupling that naturally exists between various axes of a piezoelectric tube scanner. However, experimental results confirm that this cross-coupling could be substantial. A crosscoupling of $20 \mathrm{~dB}$ at low frequencies is quite normal, and this effect could be even more severe at the resonance frequency of the tube. ${ }^{72}$ That is, a slow $10 \mu \mathrm{m}$ triangular motion of one axis can translate into a $1 \mu \mathrm{m}$ triangular motion of the other axis, generating substantial distortion in the resulting image. This effect becomes more significant if the actuator is used in a high-speed scanning regime. ${ }^{73}$ While 


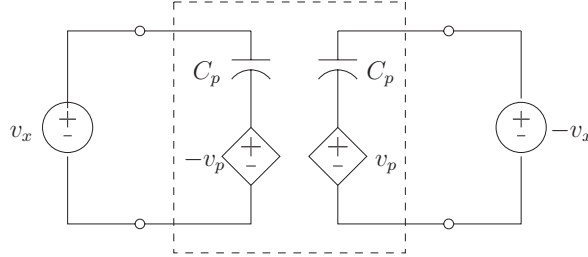

(a)

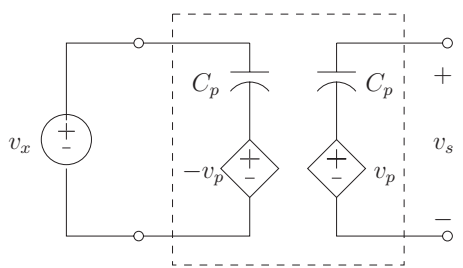

(b)

FIG. 7. Electrical circuit representing a piezoelectric tube scanner under (a) symmetrical and (b) asymmetrical actuations.

cross-coupling is an inherent property of piezoelectric tube actuators, the eccentricity of the tube is known to exacerbate its effect. ${ }^{66}$ Feedback can be used to address problems of this nature. This awareness has contributed to significant studies aimed at developing a good understanding of the dynamics of piezoelectric tube actuators (see Refs. 74-79 and references therein).

\section{ELECTRICAL MODEL OF A PIEZOELECTRIC TUBE}

From an electrical point of view, each electrode of a piezoelectric tube can be modeled as a capacitor in series with a dependent voltage source. The existence of this voltage is an immediate consequence of the piezoelectric effect and is directly related to the mechanical strain experienced by each quarter of the tube. ${ }^{80,81}$ Figure 7 (a) illustrates the electrical equivalent of the symmetrically driven actuator illustrated in Fig. 2(a). The key assumption here is that the tube is perfectly symmetrical and uniform. Notice that the piezoelectric voltages induced in the opposite electrodes are $180^{\circ}$ out of phase. This reflects the fact that while one side of the tube is extended, the other side is equally contracted. Furthermore, this implies that the polarization vector points radially outward.

The electrical equivalent of the asymmetrically driven tube in Fig. 2(b) is depicted in Fig. 7(b). The assumption made here is that both sides of the tube undergo equal, but opposite, strains, and consequently the piezoelectric voltages induced in the opposite electrodes are equal, but $180^{\circ}$ out of phase. Although, in practice, there is a small difference between these two voltages (due to the asymmetric nature of actuation), for most applications this approximation is quite acceptable. Also, notice that for identical applied voltages, the $v_{p}$ corresponding to the asymmetrical case is half of that when the tube is driven symmetrically. The situation depicted in Fig. 2(b) presents us with a possibility that until very recently (and rather surprisingly) appears to have escaped the attention of most researchers. The free electrode can be used as a sensor.

The frequency response of the tube when one of the electrodes is driven by a signal and the voltage induced in
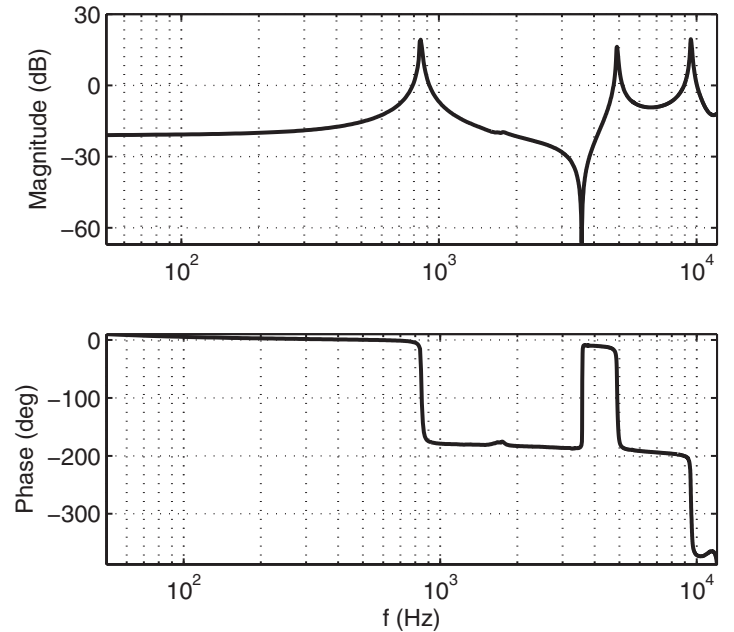

FIG. 8. Frequency response of a piezoelectric tube scanner with quartered external electrodes and a single inner electrode. One of the electrodes is taken as the input. The voltage induced in the opposite electrode, due to the piezoelectric effect, is measured directly by a spectrum analyzer over a bandwidth of $10 \mathrm{kHz}$. Note that the first two poles of this transfer function are identical to the displacement frequency response of the tube in Fig. 3. However, the two transfer functions have different zeros. Moreover, the third mode that is missing from Fig. 3 is the piston mode of the tube.

the opposite electrode is taken as the output is plotted in Fig. 8. Comparing this with the displacement frequency response of the same scanner in Fig. 3, one can make a number of interesting and important observations. Both systems have identical resonance frequencies; i.e., the two transfer functions have "identical poles." The third resonance in Fig. 3 appears to have an insignificant magnitude. However, the phase transition clearly illustrates its existence. Incidentally, the third mode is the "piston mode" of the scanner, which is expected to have a very low profile on its lateral displacement frequency response. In most nanoscale positioning applications, the tube is operated at frequencies well below its first resonance frequency. Over these range of frequencies, the piezoelectric voltage induced in the free electrode, $v_{p}$, can be used as a measure of lateral deflection of the tube. This is illustrated in Fig. 9, which compares the two frequency responses from $1 \mathrm{~Hz}$ to $1 \mathrm{kHz}$. It can be observed that between $10 \mathrm{~Hz}$ and the first resonance frequency of the tube, the two frequency response functions are almost identical. At frequencies below $10 \mathrm{~Hz}$, however, the frequency response function (FRF) corresponding to $v_{p}$ is clearly distorted. This is due to the fact that $v_{p}$ is measured by a device that has a finite input impedance. Assuming that the measurement instrument has an input resistance of $R_{\text {in }}$, the measured voltage, $v_{s}$, is

$$
v_{s}=\frac{s}{s+\tau},
$$

where $\tau=1 / R_{\text {in }} C_{p}$. Thus, the signal measured at the open terminal of the tube will be distorted at low frequencies and will not be able to determine the static deflection of the tube. The low-frequency nature of the measured signal can be substantially improved if a buffer circuit with a very high input impedance is introduced between the free electrode and the measurement instrument. Although the input impedance of 

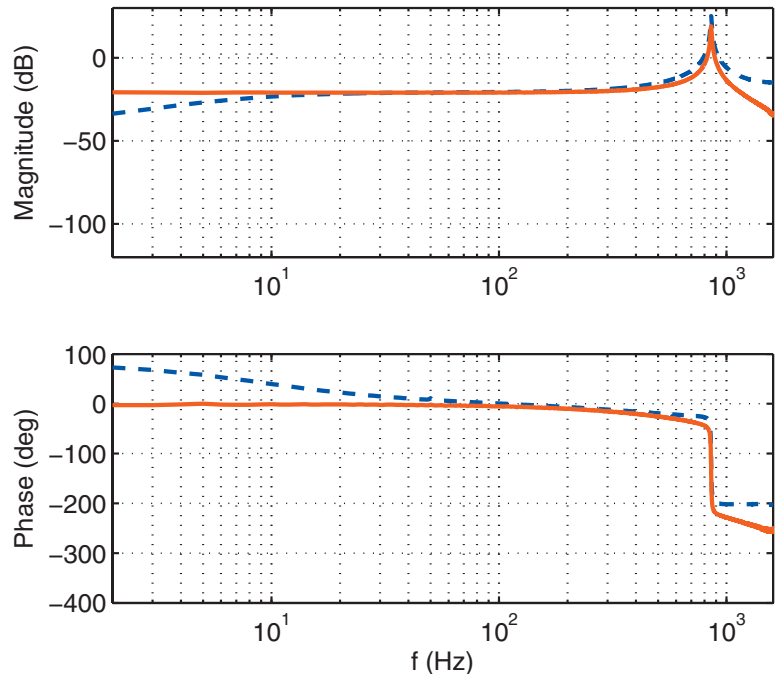

FIG. 9. (Color) The two frequency responses represent the lateral deflection of the free end of the tube measured by a capacitive sensor $(-)$ and the piezoelectric voltage induced in the opposite electrode (--).

the buffer can be made extremely large, it will always be a finite number. Thus, the problem at dc remains. The problem is further compounded due to the existence of dielectric leakage current in the sensing electrode, although the parallel resistor introduced corresponding to this effect is often quite large.

\section{A. Use of piezoelectric voltage in a feedback loop}

Despite its poor low-frequency nature, the piezoelectric voltage can be used for a number of purposes. An immediate possibility is to use it in a feedback loop to augment the tube's damping. This idea was recently implemented in Refs. 82 and 72 with noticeable success. In this approach, the piezoelectric voltage is used as a measurement, and a controller is designed to add substantial damping to the first resonant mode of the tube. This flattens the tube's frequency response, and the better damped scanner is then driven by a triangular signal for rastering. Due to its flatter frequency response, the scanner can be used at much higher speeds; i.e., faster triangular waveforms can be utilized. Thus, in this approach, the main purpose of the controller is to better damp the tube. Once this is achieved, the better damped scanner is essentially driven in an open loop.

To use the piezoelectric voltage as a measurement signal is a quite sensible option, particularly considering its compelling noise properties. The rms displacement noise of the scanner described in Sec. III was measured to be in the order of $16 \mathrm{fm} / \sqrt{\mathrm{Hz}}^{83,84}$ When compared with the rms noise corresponding to the capacitive sensor measurement described in Sec. III E, i.e., $20 \mathrm{pm} / \sqrt{\mathrm{Hz}}$, this shows a three order of magnitude improvement, assuming that both sensors are operated over the same bandwidth. However, since the piezoelectric displacement sensor cannot be used for dc and lowfrequency measurements, the controller cannot be used to address issues such as creep and thermal drift, nor can it be used for accurate dc positioning.

The above discussion raises an intriguing possibility: a capacitive sensor can be used for dc and low-frequency dis-

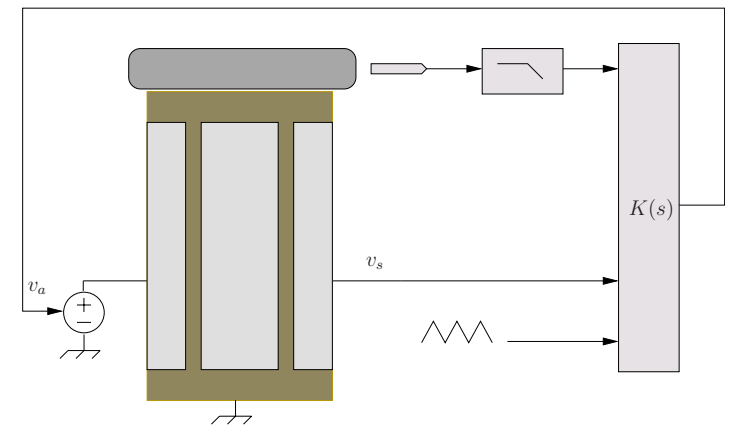

FIG. 10. (Color online) Schematics of a two-sensor-based tracking controller for a piezoelectric tube scanner. The capacitive sensor's signal is low pass filtered to reduce its stochastic noise component, thus limiting its use to dc and low frequencies where the piezoelectric displacement signal is not reliable. The controller is designed to track a reference signal illustrated as a triangular waveform.

placement measurements, while the piezoelectric signal can be employed to measure the same quantity, but over the frequencies that lie out of the bandwidth of the capacitive sensor. This arrangement would enable one to lower the bandwidth of the capacitive sensor and, subsequently, to increase its sensitivity. The signals produced by the two sensors would have to be processed in a manner suitable for feedback control. Two possibilities have been recently investigated. In Refs. 85 and 86 a two-sensor-based $H_{\infty}$ controller was designed directly using the two complementary displacement signals. In this approach, the two-input, oneoutput controller uses the capacitive sensor measurement at low frequencies, through to the dc, and for high-frequency tracking and damping of the resonance frequency it uses the piezoelectric voltage signal. This situation is sketched in Fig. 10. The implementation of this technique on the piezoelectric tube scanner whose open-loop responses are plotted in Fig. 5 results in closed-loop responses illustrated in Fig. 11. A significant improvement is clearly observable. An alternative approach, presented in Refs. 83 and 84, is to first generate an optimal estimate of the displacement using the two complementary signals and then to design a single-input singleoutput (SISO) controller that utilizes this estimate to achieve the necessary tracking. The standard tool for such "sensor

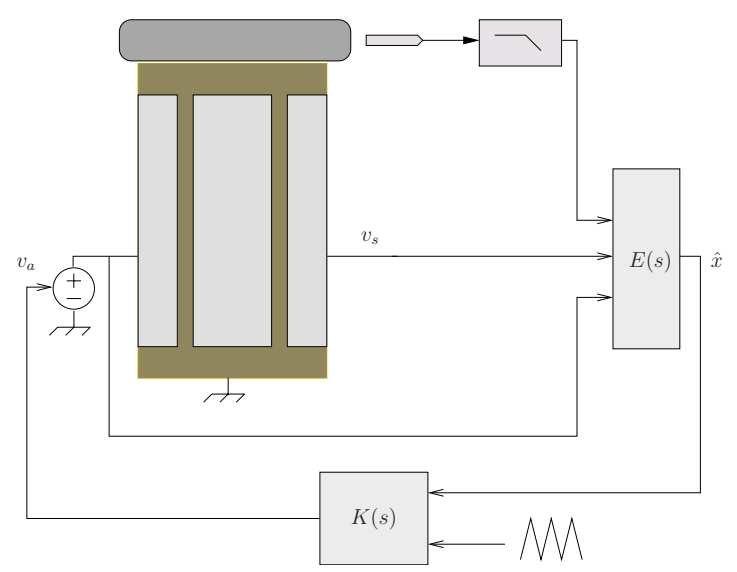

FIG. 11. (Color online) Closed-loop lateral movement of a piezoelectric tube scanner when driven by (a) $5 \mathrm{~Hz}$, (b) $20 \mathrm{~Hz}$, and (c) $40 \mathrm{~Hz}$ signals. 


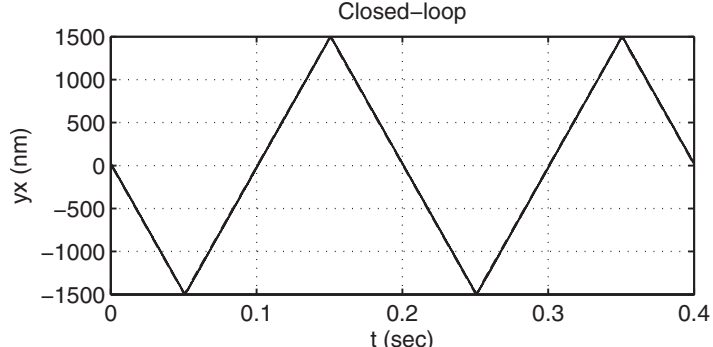

(a)

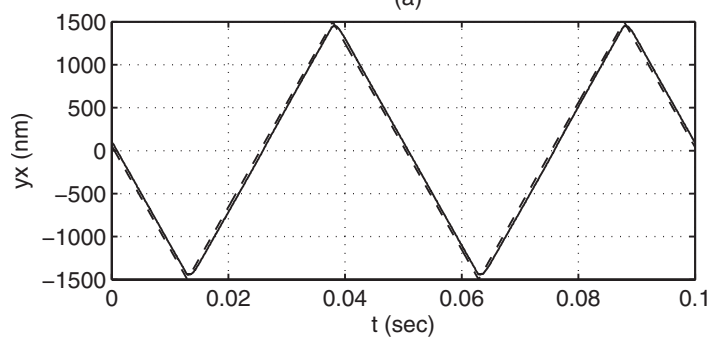

(b)

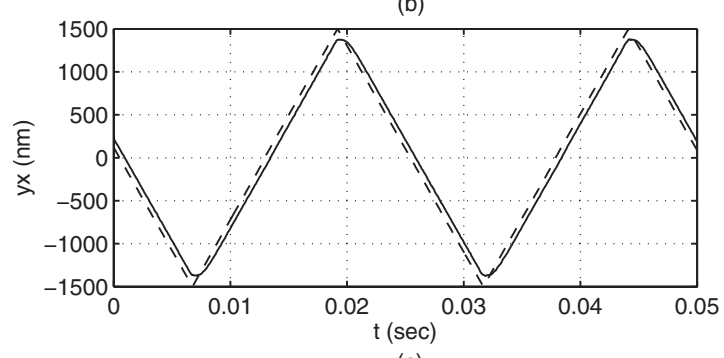

(c)

FIG. 12. An alternative approach to the arrangement in Fig. 10. The two complementary sensor signals are "fused" together using a Kalman filter. The optimal estimate of the position is then used for feedback.

fusion" is the Kalman filter. ${ }^{87}$ This arrangement is depicted in Figure 12.

\section{B. Sensorless control of a tube}

Having access to the piezoelectric voltage induced in the free electrode of a tube enables one to augment the structural damping of the system by shunting the free electrode to an impedance. The idea is illustrated in Fig. 13, where the free electrode is shunted to an $R L$ impedance. In this arrangement the inductor $L$ and the piezoelectric capacitor $C_{p}$ are tuned to the first resonance frequency of the tube, $\omega$, i.e.,

$$
L=\frac{1}{\omega^{2} C_{p}} .
$$

The resistor $R$ is then chosen such that damping of the shunted system is maximized.

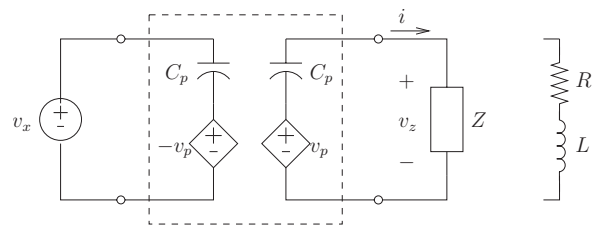

FIG. 13. Electrical equivalent of a piezoelectric tube scanner. One side of the tube is driven by the tracking signal while the opposite side is shunted to an impedance. An $L R$ impedance, when tuned to the tube's first resonance frequency, is known to result in a better damped system.

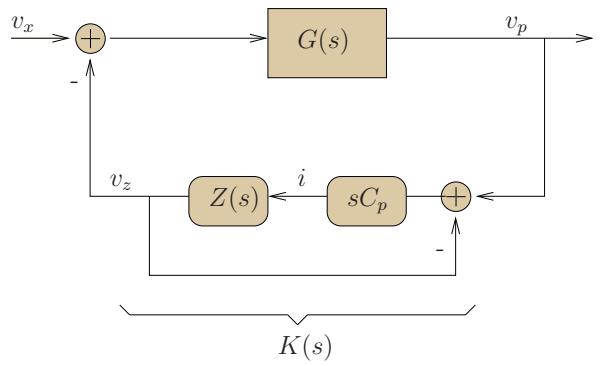

FIG. 14. (Color online) Feedback structure of a shunted piezoelectric tube scanner. Note that the shunted tube is equivalent to a collocated tube under the feedback controller (8).

The idea of piezoelectric shunt control was initially introduced in Ref. 88 and later revisited in Ref. 89 in more depth. Ever since, this technique has been widely used in vibration control applications with piezoelectric actuators (see Refs. 90 and 91 and references therein). Although piezoelectric actuators are the most widely used means of actuation in nanoscale positioning systems, this approach has not been used in this context until very recently.

To understand the operation of a shunted piezoelectric tube actuator, consider the situation depicted in Fig. 13, where the electrode located on one side of the tube is driven by the tracking signal, $v_{x}$, and the opposite electrode is shunted to an impedance, $Z$. The three equations that describe the system are

$$
\begin{aligned}
& v_{p}=G(s) v_{x}-G(s) v_{z}, \\
& i=C_{p} s\left(v_{p}-v_{z}\right), \\
& v_{z}=Z(s) i .
\end{aligned}
$$

The first equation is a direct consequence of the piezoelectric effect and represents the immediate impact of the impedance on the dynamics of the tube. Here, $G(s)$ is the collocated transfer function of the tube that describes the highly resonant nature of the structure. This model can be easily identified. The second equation results from the KVL, written around the shunted loop, and the third equation follows from Ohm's law. Equations (5)-(7) can be visualized in a block diagram form, as illustrated in Fig. 14. An inspection of this block diagram reveals that the presence of the impedance, $Z(s)$, amounts to a feedback controller described by

$$
K(s)=\frac{C_{p} s Z(s)}{1+C_{p} s Z(s)}
$$

wrapped around the plant $G(s)$. This is an interesting and important observation: instead of using one electrode as a sensor and the opposite electrode as an actuator, we may integrate both functionalities into the very same electrode. Therefore, this method enables us to utilize both electrodes, simultaneously, for sensing and actuation. Consequently, the scanning range of the scanner can be doubled. This is illustrated in Fig. 15, where the requisite impedance is implemented on one of the electrodes, and the voltage at that electrode is measured, inverted, and then applied to the opposite electrode. Furthermore, if the impedance is chosen to be as 


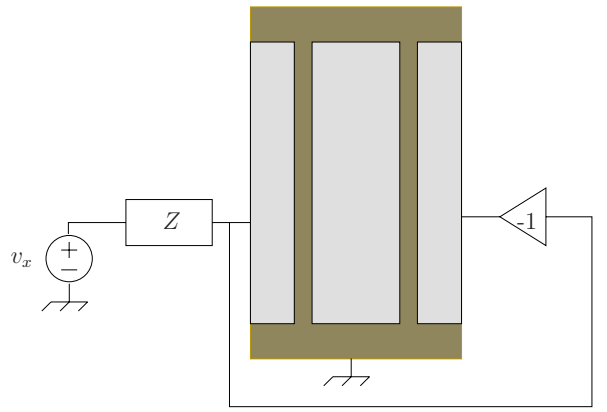

FIG. 15. (Color online) Both sides of a piezoelectric tube scanner can be used simultaneously for actuation and damping. The signal applied to the shunted electrode is inverted, using an inverting amplifier with a unity gain, and applied to the opposite electrode.

shown in Fig. 13, i.e., $Z(s)=R+L s$, the controller turns out to be

$$
K(s)=\frac{s\left(s+\frac{R}{L}\right)}{s^{2}+\frac{R}{L} s+\frac{1}{L C_{p}}} .
$$

This is the most simple form of a resonant controller described in Refs. 92-94. The controller is perfectly suited to add damping to the structure, and it has important robustness properties described in the above references. The implementation of an $R L$ impedance, however, may not be as straightforward as one would wish. The main complication is due to the highly capacitive nature of the tube and its relatively low first resonance frequency. The piezoelectric capacitance of the tube, $C_{p}$, is about $22 \mathrm{nF}$. Given that the tube's first resonance frequency is approximately at $850 \mathrm{~Hz}$, the requisite inductance is found to be about $1.6 \mathrm{H}$, clearly difficult to realize in practice. The synthetic impedance described in Ref. 95 is a practical way of implementing impedances of this nature. An important advantage of this approach is that any impedance can be implemented digitally.

The resistive-inductive shunt proposed in Ref. 89 is known to result in good damping when applied to a piezoelectric transducer, including a piezoelectric tube scanner. ${ }^{46}$ However, due to its passive nature, the maximum damping that such an impedance can generate is limited. To achieve a higher level of damping, an "active" impedance can be designed and implemented digitally as a synthetic impedance. ${ }^{95}$ Having identified the feedback structure associated with a shunted piezoelectric tube scanner (Fig. 14) enables one to treat the impedance as a feedback controller and the remainder of the system as an augmented plant. A variety of control design techniques can then be used to achieve the damping needed to increase the scanning speed of the apparatus. This procedure was applied recently to a piezoelectric tube scanner in Ref. 96, where $\mathrm{H}_{2}$ and $H_{\infty}$ optimal impedances were implemented on a prototype nanopositioner with very good success.

A very attractive feature of the procedure described above is how easily it can be incorporated into the currently available SPMs. Majority of SPMs use piezoelectric tube scanners for rastering. All of these tubes have quartered ex- ternal electrodes that can be used for simultaneous actuation and sensing. Instrumenting a tube with an appropriate impedance can be achieved by making simple modifications to the electronic driving module. Digital implementation of the impedance can be realized using the computer that is often an integral part of any SPM. Furthermore, this approach does not require any changes to the mechanical components of the SPM; e.g., there is no need to introduce displacement sensors into the scanning head of the SPM.

Before concluding this section, we reiterate that the best way to actuate piezoelectric tube scanners is to do it with a charge source. However, since driving piezoelectric actuators with voltage amplifiers is more common, in our presentation, we have opted to assume that the piezoelectric scanner is actuated with a voltage amplifier. Further details on charge driven piezoelectric tube actuators can be found in Ref. 46.

\section{FUTURE CHALLENGES AND CONCLUSION}

Piezoelectric tube scanners are an integral part of modern SPMs. To achieve a fast and accurate operation of a SPM, one is required to address the issues that hinder the positioning accuracy and bandwidth of its scanner. This paper presented a survey of a number of recently emerged technologies and methodologies aimed at maximizing the scanning range, precision, and scanning speed of piezoelectric tube scanners. To this end it should be clear that over the past two decades a significant volume of research has appeared on piezoelectric tube scanners. This may falsely lead one to conclude that "what could have been done has already been done." However, as the preceding discussion made clear, significant improvements can be achieved by utilizing advanced feedback control and estimation techniques and innovative ideas and methods that have emerged in other fields, e.g., smart structures. By combining these techniques, a significant improvement in the operating conditions of a scanner can be achieved. This will, in turn, translate into faster and more accurate SPMs.

Real-time implementation of these methods often requires access to the fast digital signal processing hardware and the requisite software. For small tubes, with the first resonance frequency in the tens (or hundreds) of kilohertz, such implementations remain quite challenging. Analog control implementation could be an answer, although one would need to address a significant number of issues that naturally arise in such implementations. Field programmable gate array and its derivative technologies can also be used for this purpose.

An important matter that has escaped the attention of researchers in this field is the possibility of designing alternative electrode patterns for piezoelectric tube scanners. Lateral positioning is typically achieved by a tube with quartered external electrodes. There is no reason why one should limit oneself to the "traditional" electrode pattern to achieve lateral actuation. It should be possible to pattern the external electrodes to achieve satisfactory lateral movements and simultaneous sensing. Also, it may be possible to shape the 
electrodes such that only the required modes of the tube are excited if one uses the orthogonal properties that govern the mode shapes of a tube.

To conclude, piezoelectric tube scanners are currently the most widely utilized nanopositioning tool. They are easily manufactured, widely available, and inexpensive. Their widespread use is likely to continue well into the future. Despite their simple construction, these scanners are capable of providing astonishing positioning accuracy over notable bandwidths. Both the positioning accuracy and the bandwidth of piezoelectric tube scanners can be substantially improved by employing model-based control and estimation techniques together with a deeper understanding of properties of piezoelectric materials, of which these devices are made. This approach to nanopositioning is likely to result in interesting applications that may be difficult to envision at the time of this writing. Nonetheless, we are set to witness interesting progress in this field in the near future.

\section{ACKNOWLEDGMENTS}

The author wishes to thank the Australian Research Council and the Centre for Complex Dynamic Systems and Control for supporting his research that underlies this review. He also wishes to thank all of his colleagues at the Laboratory for Dynamics and Control of Nanosystems, particularly Iskandar Mahmood for his help with this work.

${ }^{1}$ G. Binnig and H. Rohrer, Helv. Phys. Acta 55, 726 (1982).

${ }^{2}$ G. Binnig, H. Rohrer, C. Gerber, and E. Weibel, Phys. Rev. Lett. 49, 57 (1982).

${ }^{3}$ I. Giaever, Phys. Rev. Lett. 5, 147 (1960).

${ }^{4}$ G. Binnig, C. F. Quate, and C. Gerber, Phys. Rev. Lett. 56, 930 (1986).

${ }^{5}$ P. K. Hansma and J. Tersoff, J. Appl. Phys. 61, 1 (1987).

${ }^{6}$ D. Sarid and V. Elings, J. Vac. Sci. Technol. B 9, 431 (1991).

${ }^{7}$ J. Frommer, Angew. Chem., Int. Ed. Engl. 31, 1298 (1992).

${ }^{8}$ Springer Handbook of Nanotechnology, edited by B. Bhushan (Springer, Berlin, Germany, 2004).

${ }^{9}$ G. Binnig and D. P. E. Smith, Rev. Sci. Instrum. 57, 1688 (1986).

${ }^{10}$ See A. Research, http://www.asylumresearch.com/ for more information on use of flexure-based positioners in AFMs.

${ }^{11}$ S. Aphale, B. Bhikkaji, and S. O. R. Moheimani, IEEE Trans. Nanotechnol. 7, 79 (2008).

${ }^{12}$ See PI Ceramics, http://www.piceramic.com/ for more information on flexure-based positioning systems.

${ }^{13}$ S. Salapaka, A. Sebastian, J. P. Cleveland, and M. V. Salapaka, Rev. Sci. Instrum. 73, 3232 (2002).

${ }^{14}$ S. Salapaka, A. Sebastian, J. P. Cleveland, and M. V. Salappaka, Proceedings of the American Control Conference, 2002 (unpublished), pp. 1966-1971.

${ }^{15}$ A. Sebastian and S. M. Salapaka, IEEE Trans. Control Syst. Technol. 13, 868 (2005)

${ }^{16}$ T. Ando, N. Kodera, D. Maruyama, E. Takai, K. Saito, and A. Toda, Jpn. J. Appl. Phys., Part 1 41, 4851 (2002).

${ }^{17}$ N. Kodera, H. Yamashita, and T. Ando, Rev. Sci. Instrum. 76, 1 (2005).

${ }^{18}$ J. S. Yang and H. Y. Fang, Sens. Actuators, A 107, 42 (2003).

${ }^{19}$ Y. Kagawa, N. Wakatsuki, T. Tsuchiya, and Y. Terada, Inf. Econ. Policy 6, 325 (2006)

${ }^{20}$ S. Chao, J. L. Garbini, W. M. Dougherty, and J. A. Sidles, Rev. Sci. Instrum. 77,063710 (2006).

${ }^{21}$ A. S. Putra, S. Huang, K. K. Tan, S. K. Panda, and T. H. Lee, IEEE Trans. Control Syst. Technol. 15, 879 (2007).

${ }^{22}$ R. G. Carr, J. Microsc. 152, 379 (1988)

${ }^{23}$ C. J. Chen, Ultramicroscopy 42, 1653 (1992).

${ }^{24}$ C. J. Chen, Appl. Phys. Lett. 60, 132 (1992).

${ }^{25}$ Q. Sun and R. A. Wolkow, Rev. Sci. Instrum. 77, 113701 (2006).

${ }^{26}$ J. Maess, A. J. Fleming, and F. Allgower, Rev. Sci. Instrum. 79 015105(2008).
${ }^{27}$ B. P. Lathi, Linear Systems and Signals, 2nd ed. (Oxford University Press, New York, 2004).

${ }^{28}$ E. Meyer, H. J. Hug, and R. Bennewitz, Scanning Probe Microscopy (Springer, Heidelberg, Germany, 2004).

${ }^{29}$ I. Mayergoyz, Mathematical Models of Hysteresis (Springer, New York, 1991).

${ }^{30}$ M. Goldfarb and N. Celanovic, J. Dyn. Syst., Meas., Control 119, 478 (1997).

${ }^{31}$ P. Ge and M. Jouaneh, Precis. Eng. 17, 211 (1995).

${ }^{32}$ R. B. Mrad and H. Hu, IEEE/ASME Trans. Mechatron. 7, 479 (2002).

${ }^{33}$ S. H. Lee and T. J. Royston, J. Acoust. Soc. Am. 108, 2843 (2000).

${ }^{34} \mathrm{~J}$. Zhong and B. Yao (unpublished).

${ }^{35}$ B. M. Chen, T. H. Lee, H. Chang-Chieh, Y. Guo, and S. Weerasooriya, IEEE Trans. Control Syst. Technol. 7, 160 (1999).

${ }^{36}$ J. M. Cruz-Hernandez and V. Hayward, IEEE International Conference on Robotics and Automation, 1998 (unpublished), pp. 1510-1515.

${ }^{37}$ J. M. Cruz-Hernandez and V. Hayward, IEEE Trans. Control Syst. Technol. 9, 17 (2001).

${ }^{38}$ J. A. Main and E. Garcia, J. Guid. Control Dyn. 20, 327 (1997).

${ }^{39}$ J. A. Main and E. Garcia, J. Guid. Control Dyn. 20, 479 (1997).

${ }^{40}$ R. Comstock, U.S. Patent No. 4,263,527 (1981).

${ }^{41}$ C. Newcomb and I. Flinn, Electron. Lett. 18, 442 (1982).

${ }^{42}$ J. A. Main, E. Garcia, and D. V. Newton, J. Guid. Control Dyn. 18, 1068 (1995).

${ }^{43}$ A. J. Fleming and S. O. R. Moheimani, Electron. Lett. 39, 282 (2003).

${ }^{44}$ A. J. Fleming and S. O. R. Moheimani, J. Intell. Mater. Syst. Struct. 15, 77 (2004).

${ }^{45}$ A. J. Fleming and S. O. R. Moheimani, Rev. Sci. Instrum. 76, 073707 (2005)

${ }^{46}$ A. J. Fleming and S. O. R. Moheimani, IEEE Trans. Control Syst. Technol. 14, 33 (2006).

${ }^{47} \mathrm{~K}$. Yi and R. J. Veillette, IEEE Trans. Control Syst. Technol. 13, 517 (2005).

${ }^{48}$ C. Ru, L. Chen, B. Shao, W. Rong, and L. Sun, Meas. Sci. Technol. 19, 015203 (2008).

${ }^{49}$ T. Fett and G. Thun, J. Mater. Sci. Lett. 17, 1929 (1998).

${ }^{50}$ R. S. Robinson, J. Comput.-Assist. Microsc. 2, 53 (1996).

${ }^{51}$ H. Jung, J. Y. Shim, and D. Gweon, Rev. Sci. Instrum. 71, 3436 (2000).

${ }^{52}$ D. Croft, G. Shed, and S. Devasia, J. Dyn. Syst., Meas., Control 123, 35 (2001).

${ }^{53}$ A. A. Tsenga, A. Notargiacomob, and T. P. Chen, J. Vac. Sci. Technol. A 23, 877 (2005).

${ }^{54}$ B. D. Gates, Q. Xu, J. C. Love, D. B. Wolfe, and G. M. Whitesides, Annu. Rev. Mater. Res. 34, 339372 (2004).

${ }^{55}$ F. J. R.-S. W. M. Heckle and R. W. Stark, Adv. Eng. Mater. 7, 193 (2005).

${ }^{56}$ K. J. G. Hinnen, R. Fraanje, and M. Verhaegen, Journal of Systems and Control Engineering 218, 503 (2004).

${ }^{57}$ W. Vogl, B. K.-L. Ma, and M. Sitti, IEEE Trans. Nanotechnol. 5, 397406 (2006).

${ }^{58}$ B. Mokaberi and A. G. Requicha, IEEE. Trans. Autom. Sci. Eng. 3, 3 (2006).

${ }^{59}$ V. Y. Yurov and A. N. Klimov, Rev. Sci. Instrum. 65, 1551 (1994).

${ }^{60}$ R. Staub, D. Alliata, and C. Nicoloini, Rev. Sci. Instrum. 66, 2513 (1995).

${ }^{61}$ J. T. Woodward and D. K. Schwartz, J. Vac. Sci. Technol. B 16, 51 (1998).

${ }^{62}$ K. J. Ito, Y. Uehara, S. Ushioda, and K. Ito, Rev. Sci. Instrum. 71, 420 (2000).

${ }^{63}$ S. H. Huerth and H. D. Hallen, J. Vac. Sci. Technol. B 21, 714 (2003).

${ }^{64} \mathrm{~N}$. Tamer and M. Dahleh, Proceedings of the 33rd IEEE Conference on Decision and Control, Lake Buena Vista, FL, 1994 (unpublished), pp. $1826-1831$.

${ }^{65}$ A. Daniele, S. Salapaka, M. V. Salapaka, and M. Dahleh, Proceedings of the American Control Conference, 1999 (unpublished), pp. 253-257.

${ }^{66}$ O. M. E. Rifai and K. Youcef-Tomi, Proceedings of the American Control Conference, Arlington, VA, 2001 (unpublished), pp. 3251-3255.

${ }^{67}$ G. Schitter and A. Stemmer, IEEE Trans. Control Syst. Technol. 12, 449 (2004)

${ }^{68}$ G. Schitter, P. Menold, H. F. Knapp, F. Allgower, and A. Stemmer, Rev. Sci. Instrum. 72, 3320 (2001).

${ }^{69}$ R. C. Barrett and C. F. Quate, Rev. Sci. Instrum. 62, 1393 (1991).

${ }^{70}$ K. Leang and S. Devasia, Second IFAC Conference on Mechatronic Systems, Berkeley, CA, 2002 (unpublished), pp. 283-289.

${ }^{71}$ D. Croft, G. Shed and S. Devasia, J. Dyn. Syst., Meas., Control 123, 35 (2001).

${ }^{72}$ B. Bhikkaji, M. Ratnam, A. J. Fleming, and S. O. R. Moheimani, IEEE 
Trans. Control Syst. Technol. 5, 853 (2007).

${ }^{73}$ S. Tien, Q. Zou, and S. Devasia, IEEE Trans. Control Syst. Technol. 13, 921 (2005)

${ }^{74}$ M. E. Taylor, Rev. Sci. Instrum. 64, 154 (1993).

${ }^{75}$ T. Ohara and K. Youcef-Toumi, Proceedings of the American Control Conference, Seattle, Washington, 1995 (unpublished), pp. 3808-3812.

${ }^{76}$ S. Yang and W. Huang, Rev. Sci. Instrum. 68, 4483 (1997).

${ }^{77}$ J. Tapson and J. R. Greene, Rev. Sci. Instrum. 68, 2797 (1997).

${ }^{78}$ S. Yang and W. Huang, Rev. Sci. Instrum. 69, 226 (1998).

${ }^{79}$ S. Bonnail, D. Tonneau, G. A. Capolino, and H. Dallaporta, Proceedings of the IEEE Industry Applications Conference, 2000 (unpublished), Vol. 1, pp. 293-298.

${ }^{80}$ Institute of Electrical and Electronics Enginners Inc., IEEE Standard on Piezoelectricity, ANSI/IEEE Std. No. 176-1987, 1988.

${ }^{81}$ T. Ikeda, Fundamentals of Piezoelectricity (Oxford University Press, New York, 1996).

${ }^{82}$ B. Bhikkaji, M. Ratnam, and S. O. R. Moheimani, Sens. Actuators, A 135, 700 (2007).

${ }^{83}$ A. J. Fleming, A. Wills, and S. O. R. Moheimani, IEEE Trans. Control Syst. Technol., DOI:10.1109/TCST.2008.921798.

${ }^{84}$ S. Aphale, A. J. Fleming, and S. O. R. Moheimani, Proceedings of the
American Control Conference, New York, 2007 (unpublished)

${ }^{85}$ I. A. Mahmood, S. O. R. Moheimani, and K. Liu IEEE Trans. Nanotechnol. (unpublished)

${ }^{86}$ I. A. Mahmood, S. O. R. Moheimani, and K. Liu, Proceedings of the 17 th IFAC World Congress, 2008 (unpublished).

${ }^{87}$ R. G. Brown and P. Y. C. Hwang, Introduction to Random Signals and Kalman Filtering (Wiley, New York, 1992), Vol. 2.

${ }^{88}$ R. L. Forward, Appl. Opt. 18, 690 (1979).

${ }^{89}$ N. W. Hagood and A. von Flotow, J. Sound Vib. 146, 243 (1991).

${ }^{90}$ G. A. Lesieutre, Shock Vib. Dig. 30, 187 (1998).

${ }^{91}$ S. O. R. Moheimani, IEEE Trans. Control Syst. Technol. 11, 482 (2003).

${ }^{92}$ D. Halim and S. O. R. Moheimani, IEEE Trans. Control Syst. Technol. 9, 37 (2001).

${ }^{93}$ H. R. Pota, S. O. R. Moheimani, and M. Smith, Smart Mater. Struct. 11, 1 (2002).

${ }^{94}$ S. Behrens, A. J. Fleming, and S. O. R. Moheimani, IEEE/ASME Trans. Mechatron. 10, 118 (2005).

${ }^{95}$ A. J. Fleming, S. Behrens, and S. O. R. Moheimani, Electron. Lett. 36, $1525(2000)$.

${ }^{96}$ S. Aphale, A. J. Fleming, and S. O. R. Moheimani, IET Micro and Nano Letters 2, 9 (2007). 